\title{
Detecção de mudanças em dados de imagens multitemporais de sensoriamento remoto empregando Support Vector Machines com o uso de kernel polinomial e kernel RBF (kernel função de base radial)
}

\author{
Rute Henrique da Silva FERREIRA ${ }^{1}$ \& Neide Pizzolato ANGELO ${ }^{2}$
}

\author{
${ }^{1}$ Curso de Matemática, Universidade La Salle. Av. Victor Barreto, 2288, CEP 92.010-000, Canoas, RS, Brasil \\ (rute.ferreira@unilasalle.edu.br). \\ ${ }^{2}$ Departamento de Matemática e Estatística / Instituto de Física e Matemática, Universidade Federal de Pelotas. Campus \\ Universitário Capão do Leão, s/no, caixa postal 354, CEP 96010-971, Pelotas, RS, Brasil (neide.angelo@gmail.com).
}

Ferreira, R.H.S., Angelo, N.P., 2018. Detecção de mudanças em dados de imagens multitemporais de sensoriamento remoto empregando Support Vector Machines com o uso de kernel polinomial e kernel RBF (kernel função de base radial). Pesquisas em Geociências, 45: e0674.

DOI: https://doi.org/10.22456/1807-9806.88649

\begin{abstract}
Resumo. Este artigo investiga uma abordagem para o problema da detecção de mudanças em imagens multitemporais de sensoriamento remoto empregando Support Vector Machines (SVM) com o uso do kernel polinomial e do kernel RBF (kernel função de base radial). Para os experimentos, foram consideradas duas imagens Landsat 5-TM cobrindo a mesma área, localizada no Estado de Roraima, Brasil $\left(61^{\circ} 37^{\prime} \mathrm{W}-61^{\circ} 49^{\prime} \mathrm{W}\right.$ de longitude e $3^{\circ} 40^{\prime} \mathrm{N}-3^{\circ} 52^{\prime} \mathrm{N}$ de latitude). A proposta metodológica está baseada na diferença de imagensfração. Em imagens de cenas naturais, a diferença nas frações de solo e vegetação tende a apresentar uma distribuição simétrica em torno da origem, fato utilizado para modelar duas distribuições normais multivariadas: mudança e não-mudança. $\mathrm{O}$ algoritmo Expectation-Maximization (EM) foi implementado para estimar os parâmetros associados a essas duas distribuições. Amostras aleatórias foram extraídas a partir das distribuições e usadas para treinar o classificador SVM. Para a avaliação da acurácia da metodologia foram utilizados dois procedimentos: a análise qualitativa, realizada por meio da produção do mapa de mudança, e a análise quantitativa, a partir da construção da matriz de confusão em uma imagem sintética. Foi possível observar que o kernel RBF apresentou resultados muito semelhantes para todos os conjuntos de amostras de teste, independentemente do tamanho do conjunto de amostras de treinamento, o que não ocorre com o kernel polinomial. Os experimentos desenvolvidos neste trabalho mostram a adequação da metodologia proposta, produzindo resultados aceitáveis na detecção de alterações na cobertura do solo, uma vez que o SVM é um método robusto, lida bem com o problema da dimensionalidade e com amostras ruidosas e necessita de um número pequeno de amostras de treinamento para o processo de classificação. Palavras-chave. Detecção de mudanças, métodos baseados em kernel, imagens-fração, algoritmo EM.
\end{abstract}

\begin{abstract}
CHANGES DETECTION IN REMOTE SENSING MULTITEMPORAL IMAGE DATA BY APPLYING SUPPORT VECTOR MACHINES WITH THE USE OF POLYNOMIAL KERNEL AND RBF KERNEL (RADIAL BASIS FUNCTION KERNEL). This paper investigates an approach to the problem of detecting changes in multitemporal remote sensing images using Support Vector Machines (SVM) with the use of the polynomial kernel and RBF kernel (kernel based radial function). For the experiments two Landsat 5-TM images were used covering the same area, located in the State of Roraima, Brazil $\left(61^{\circ} 37^{\prime} \mathrm{W}-61^{\circ} 49^{\prime} \mathrm{W}\right.$ of longitude and $3^{\circ} 40^{\prime} \mathrm{N}-3^{\circ} 52^{\prime} \mathrm{N}$ of latitude). The methodological proposal is based on the difference of fraction images. The difference in soil and vegetation fractions in natural scene images tends to have a symmetrical distribution around the origin and this fact is used to model two normal multivariate distributions: change and non-change. The Expectation-Maximization (EM) algorithm was implemented to estimate the parameters associated with these two distributions. Random samples were extracted from the distributions and used to train the SVM classifier. Two procedures were used to assess the accuracy of the methodology. First, the qualitative analysis carried out through the production of the change map. Then, the quantitative analysis carried out through the construction of the confusion matrix using a synthetic image. It was observed that the RBF kernel presented very similar results for all sets of test samples, regardless of the size of the training sample set, which does not occur with the polynomial kernel. The experiments developed in this work show the adequacy of the proposed methodology, producing acceptable results in the detection of changes in soil cover, since the SVM is a robust method, handles the problem of dimensionality and with noisy samples and requires a number small sample of training samples for the classification process.
\end{abstract}

Keywords. Change detection, kernel methods, fraction-images, EM algorithm. 


\section{Introdução}

O problema da detecção de mudanças em um conjunto de imagens da mesma cena, mas adquiridas em datas distintas, tem sido alvo de interesse em áreas como sensoriamento remoto, diagnósticos médicos, planejamento urbano e vigilância por vídeo. Em sensoriamento remoto, técnicas de detecção de mudanças em imagens multitemporais têm sido aplicadas a monitoramento agrícola, florestal, de áreas urbanas, entre outras aplicações (Zanotta \& Haertel, 2012).

$\mathrm{Na}$ literatura, duas principais abordagens para o problema da detecção de mudanças têm sido propostas na área de sensoriamento remoto: o método supervisionado e o método não supervisionado (Singh, 1989; Bruzzone \& Pietro, 2000; Lu et al., 2003; Bruzzone et al., 2004; Castellana et al., 2007; Filippone \& Sanguinetti, 2010). A primeira abordagem está baseada na análise comparativa de imagens multiespectrais cobrindo a mesma cena, mas obtidas em datas distintas. A desvantagem desse procedimento é que ele requer informação de verdade terrestre (amostras de treinamento coletadas in loco). Já na segunda abordagem, a detecção é feita pela comparação direta entre duas imagens multitemporais, sem que se faça uso de qualquer outro dado adicional. Nesse caso, o processo se dá pela aplicação de operações matemáticas e métodos estatísticos não supervisionados.

Embora o método supervisionado tenha algumas vantagens em comparação com o método não supervisionado, como a definição da natureza do tipo de mudança ocorrida, a disponibilização de amostras de treinamento é geralmente uma tarefa de difícil execução e de custo elevado. Consequentemente, o uso de métodos não supervisionados na detecção de mudanças é mais atraente em nível operacional e tem sido largamente explorado pelos pesquisadores no desenvolvimento de seus trabalhos (Zanotta \& Haertel, 2012).

As abordagens não supervisionadas para a detecção de mudanças são geralmente baseadas em imagens de diferenças, as quais são produzidas pela subtração, pixel por pixel, das imagens adquiridas em duas datas diferentes (Radke et al., 2005). As diferenças podem ser calculadas a partir dos dados originais ou a partir de dados extraídos dos originais, tais como componentes principais ou índices de vegetação. Em qualquer caso, uma imagem de diferenças é produzida e um limiar é aplicado para distinguir os pixels onde a mudança tenha ocorrido daqueles pixels que permaneceram inalterados.

Entre os vários métodos de detecção de mudança não supervisionados propostos na literatura, o mais amplamente utilizado é aquele conhecido como análise do vetor de mudanças (CVA - change vector analysis) (Malila, 1980). A técnica de CVA é baseada em três etapas: comparação da imagem por subtração de vetores, cálculo da norma dos vetores de mudança espectral (por vezes também a direção do vetor de mudança espectral é calculada) e limiarização.

Haertel et al. (2004) utilizam o conceito de pixel mistura no problema da detecção de mudanças. $\mathrm{O}$ fenômeno de mistura em pixels ocorre quando um mesmo pixel compreende duas ou mais classes distintas, denominadas, nesse contexto, de componentes (endmembers). Então é possível derivar um novo conjunto de dados com as chamadas imagens-fração, que informam em cada pixel a fração ocupada por cada uma das componentes do terreno, como, por exemplo, vegetação, solo, sombra/água.

Zanotta \& Haertel (2012) utilizam o conceito de pixel mistura para estimar com mais precisão os graus de mudanças, isto é, os graus de pertinência à classe mudança. Naquele trabalho, investigaram uma abordagem bayesiana para estimar graus de pertinência para as classes mudança e não-mudança com base nas diferenças entre as imagens-fração estimadas para duas datas diferentes.

De forma análoga, Bittencourt et al. (2013) também utilizam o conceito de pixel mistura. Entretanto, aplicam na sua abordagem estatística uma técnica multivariada para detecção de mudanças em imagens de fração, a qual fornece um limite probabilístico baseado na distribuição de qui-quadrado, no qual pixels de mudança podem ser identificados. As informações de mudança e não-mudança são então apresentadas em imagens binárias. Como meio de identificar e eliminar pixels ruidosos, operações morfológicas são aplicadas às imagens binárias. Para testar essa metodologia, 
duas imagens reais (Landsat-TM) foram empregadas, uma delas a mesma vista em Zanotta \& Haertel (2012) e no presente artigo.

Muitas abordagens propostas na literatura buscam modelar as distribuições para as classes mudança e não-mudança, a fim de estimar um valor adequado para o limiar que separa as duas classes (Bruzzone \& Pietro, 2000; Bazi et al., 2005; Bovolo et al., 2010). Por outro lado, observa-se que, nos últimos anos, classificadores baseados em kernels (núcleos) têm sido usados com sucesso em diversas áreas, como reconhecimento de faces, categorização de texto, previsão de séries temporais e reconhecimento de dígitos manuscritos. Entre eles, pode-se citar Support Vector Machines (SVM) ou Máquinas de Vetores de Suporte e as versões utilizando kernels para os métodos Análise de Componentes Principais (KPCA), Discriminante de Fisher (KFD), k-Médias e outros (Shawe-Taylor \& Cristianini, 2004).

SVM constituem um método bem adequado para se lidar com o problema da classificação de imagens hiperespectrais, uma vez que podem lidar com espaços de dimensões grandes de maneira eficiente, assim como lidam com amostras ruidosas de uma forma robusta e produzem soluções esparsas. Ou seja, o modelo que define a fronteira de decisão é expresso como uma função de um subconjunto de amostras de treinamento (Camps-Valls \& Bruzzone, 2005).

De modo semelhante ao que fora apresentado em Zanotta \& Haertel (2012) e Bittencourt et al. (2013), neste trabalho adotou-se o conceito de pixel mistura a partir do Modelo Linear de Mistura Espectral (MLME), visto que esse modelo permite que seja considerada a mistura de classes em cada pixel, a partir do uso de imagens-fração. 0 uso de imagens de fração, nesse caso, é motivado pelo fato de que elas apresentam algumas vantagens em relação às imagens espectrais. Tal abordagem apresenta duas vantagens: permite uma análise em nível de sub-pixel e elimina a necessidade de normalização radiométrica das imagens adquiridas em datas distintas, porque no processo de fracionamento são empregados pixels puros extraídos da imagem na respectiva data. Dessa forma, as imagens de diferenças são produzidas a partir da subtração de componentes gerada a partir das imagens multiespectrais. Entretanto, apesar dessa semelhança com os referidos trabalhos, que utilizam uma abordagem estatística para a classificação, este trabalho propõe o uso de Support Vector Machine (SVM), pois esse classificador, além de lidar bem com o problema da dimensionalidade e com amostras ruidosas, necessita de um número pequeno de amostras de treinamento para o processo de classificação.

Neste trabalho, o problema da detecção de mudanças foi abordado considerando inicialmente que duas distribuições gaussianas caracterizam as classes mudança e não-mudança. o algoritmo Expectation-Maximization (EM) (Duda et al., 2001) foi utilizado para estimar os parâmetros que caracterizam cada classe. Após essa etapa, fez-se uma classificação com o uso de SVM, utilizando amostras aleatórias obtidas segundo os parâmetros fornecidos pelo algoritmo EM, empregando o kernel polinomial e o kernel RBF (kernel função de base radial). Também foi realizado um experimento com dados sintéticos, com o objetivo testar o processo com um par de imagens em que se tinha um conhecimento das alterações existentes.

\section{2 Área, materiais e métodos}

\subsection{Localização da área}

Para os experimentos realizados neste trabalho, foram consideradas duas imagens Landsat 5-TM cobrindo a mesma área, localizada no Estado de Roraima, região Norte do Brasil, definida pelas coordenadas $61^{\circ} 37^{\prime} \mathrm{W}-61^{\circ} 49^{\prime} \mathrm{W}$ de longitude e $3^{\circ} 40^{\prime} \mathrm{N}-3^{\circ} 52^{\prime} \mathrm{N}$ de latitude. A área foi escolhida devido à experiência adquirida em trabalhos anteriores (Haertel et al., 2004; Zanotta, 2010; Angelo, 2014; Ferreira, 2014).

A área de estudo é caracterizada por duas planícies distintas, uma localizada a uma altitude média de $200 \mathrm{~m}$ e outra localizada em uma alta elevação, formando o Platô do Tepequém. A região de baixa elevação é coberta por uma floresta densa, enquanto a região do platô é dominada por cerrado. 0 clima na região é tropical, com uma temporada de seca bem definida (de outubro a abril) e uma estação chuvosa (de maio a setembro). A precipitação média anual varia dentro da faixa de 1750 
mm a $2000 \mathrm{~mm}$. É possível observar na literatura relatos de ocorrência de ouro e diamantes nessa região (Haertel et al., 2004), originando garimpos clandestinos.

A imagem da primeira data foi adquirida em outubro de 1991, e a imagem da segunda data, em abril de 1994. As imagens utilizadas possuem 390.625 pixels distribuídos em um quadrado de 625 por 625 pixels, conforme ilustra a figura 1 .

\subsection{Materiais e métodos}

O processo de detecção de mudanças investigado neste trabalho consiste nas seguintes etapas: pré-processamento (as imagens são registradas e as imagens-fração são produzidas); análise dos dados (produção das imagens diferença por subtração); estimação dos parâmetros da função densidade de probabilidade; produção das amostras de treinamento; classificação com SVM.
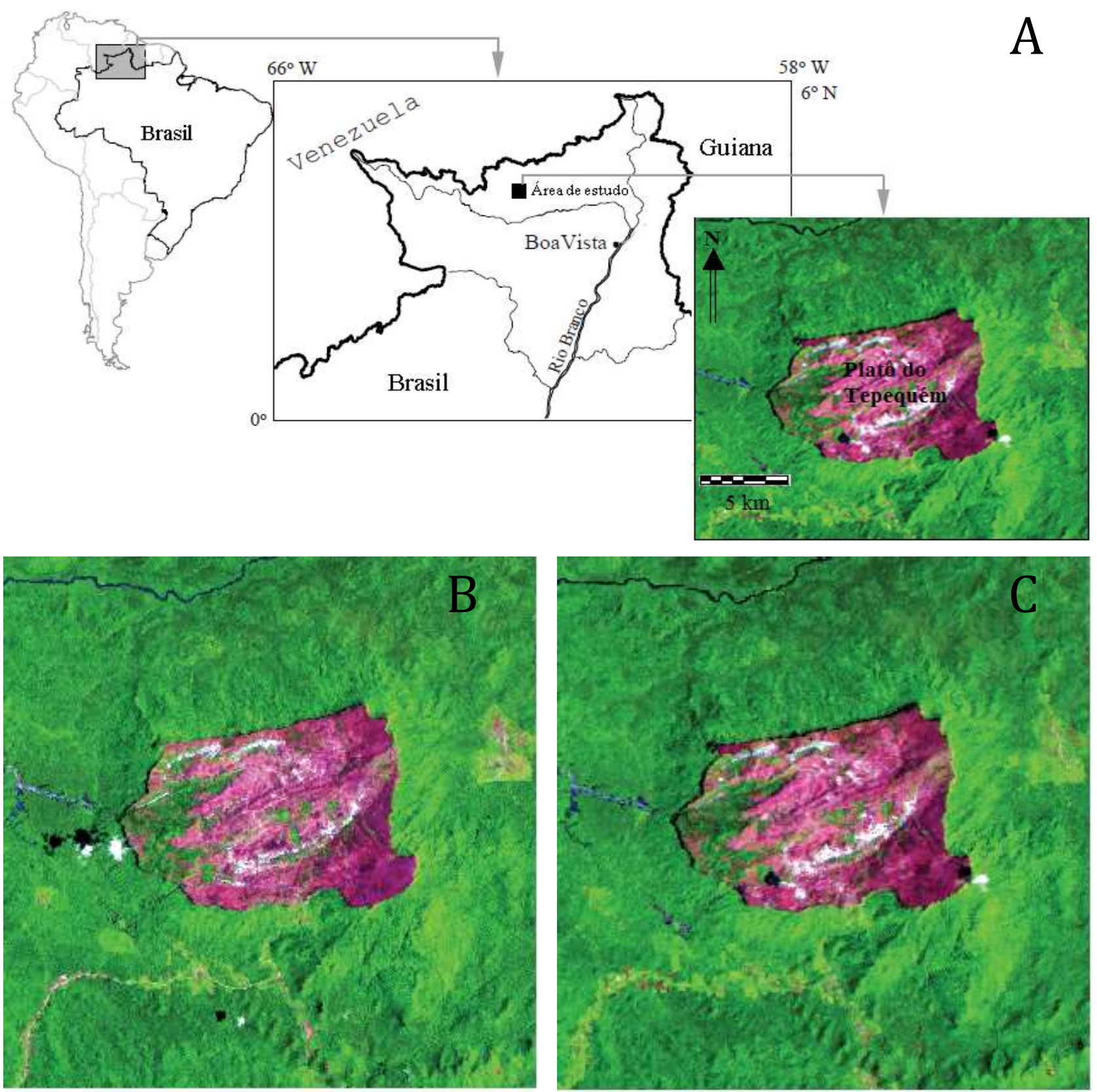

Figura 1. Localização da área de estudo e imagens utilizadas. A) Área de estudo; B) Imagem de Data 1, composição em falsa cor 5(R), 4(G), 3(B); C) Imagem de Data 2, composição em falsa cor 5(R), 4(G), 3(B) (modificado de Haertel et al., 2004).

Figure 1. Location of the study area and images used. A) Area of study; B) Image of Date 1, composition in false color 5(R), 4(G), 3(B); C) Image of Date 2, composition in false color 5(R), 4(G), 3(B) (modified from Haertel et al., 2004). 
As subseções a seguir apresentam os conceitos envolvidos em cada etapa do processo.

\subsubsection{A proposta para a abordagem do problema de detecção de mudanças}

Conforme mencionado na seção anterior, neste trabalho as imagens de diferenças são produzidas pela subtração de componentes das imagens-fração geradas a partir das imagens multiespectrais (Haertel et al., 2004).

0 método utilizado para estimar as frações das componentes de mistura (endmembers) de cada pixel da imagem é o Modelo Linear de Mistura Espectral (MLME). O MLME assume que a resposta espectral de cada pixel pode ser modelada por uma combinação linear das respostas espectrais de cada componente, ponderada pela fração da área que essa componente ocupa dentro do pixel (Shimabukuro \& Smith, 1991).

O MLME é representado por p equações lineares e $m$ frações desconhecidas, conforme equação 1.

$$
\left.R_{k}=\sum_{j=1}^{m} r_{j, k} f_{j}+e_{k}, \quad 1 \leq k \leq p \quad \text { (Equação } 1\right)
$$

Onde: as entradas do vetor Rk representam as $\mathrm{p}$ respostas espectrais dos pixels para cada banda da imagem; as entradas rj,k correspondem às respostas espectrais da componente $\mathrm{j}$ na banda $\mathrm{k}$; fj a fração do pixel ocupada pela componente j; ek representa o erro residual do modelo; p é o número de bandas espectrais em uso; e m é o número de componentes consideradas.

Para $\mathrm{p}>\mathrm{m}$ o sistema é sobredeterminado, podendo ser resolvido para as frações (fj) utilizando o método dos mínimos quadrados, sujeito às restrições expressas pela equação 2 .

$$
\forall j \quad 0 \leq f_{j} \leq 1 \quad \sum_{j=1}^{m} f_{j}=1 \quad \text { (Equação 2) }
$$

Os valores da equação devem ser selecionados de modo a representar da melhor maneira possível as componentes (endmembers) para a imagem em questão. 0 resultado do fracionamento contará com um número de imagens igual ao número de componentes escolhidas para representar a região.

Em cenas naturais, é frequente o uso das componentes vegetação, solo e sombra/água. Com base na proposta de Zanotta \& Haertel (2012), neste trabalho foram utilizadas as componentes vegetação e solo, uma vez que a componente sombra/água pode ser escrita como combinação linear das outras duas, exceto por uma pequena diferença decorrente do erro residual do modelo presente no MLME. Assim, para cada data foram geradas as imagens-fração de vegetação e solo. Esse processo foi realizado com fundamento no trabalho de Zanotta (2010), sendo implementados os algoritmos sugeridos em tal trabalho.

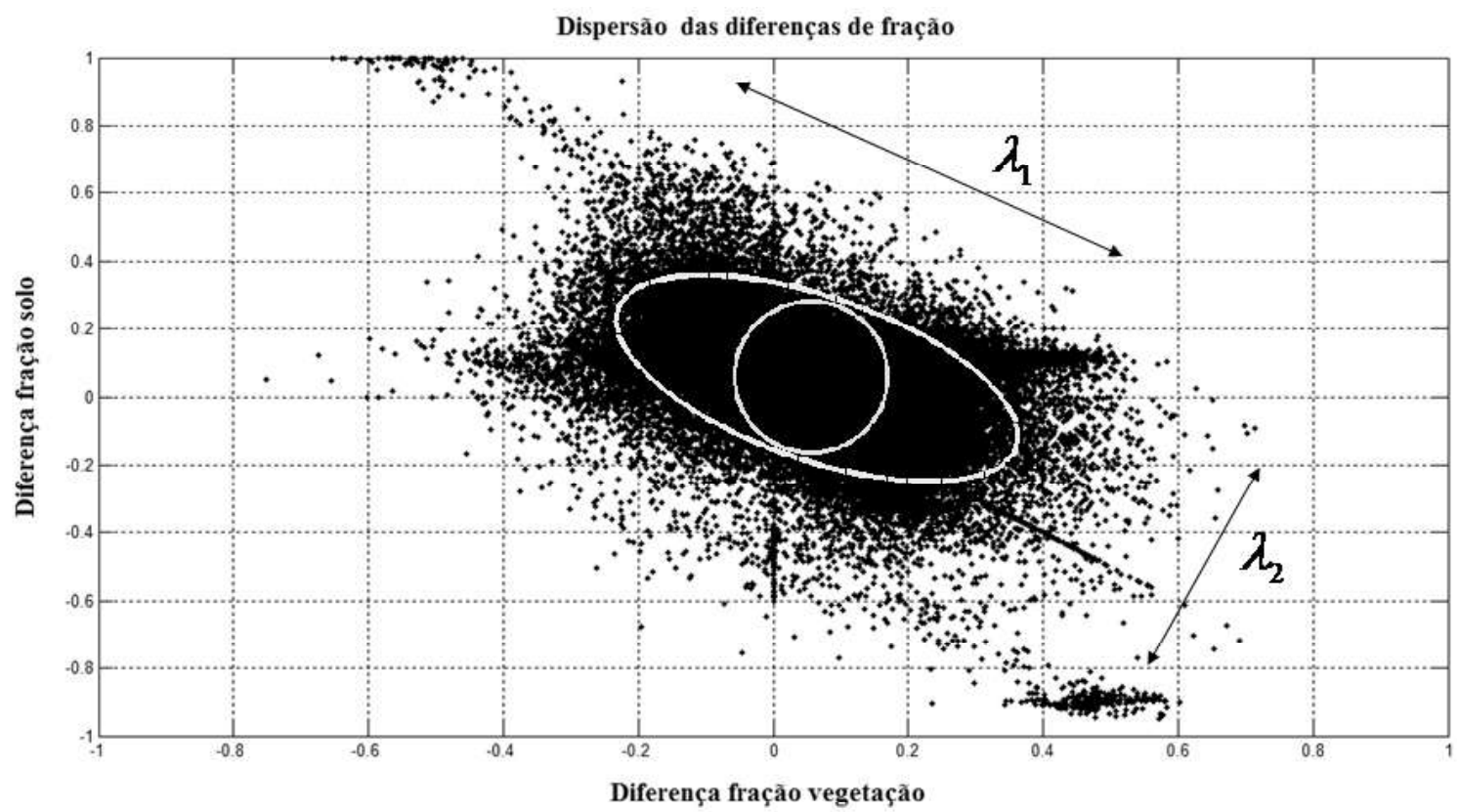

Figura 2. Espalhamento dos dados da imagem de diferenças (modificada de Zanotta, 2010).

Figure 2. Scattering of the image data of differences (modified from Zanotta, 2010). 
A figura 2 mostra o espalhamento dos dados da imagem de diferenças, considerando no eixo vertical as diferenças de solo e no horizontal as diferenças de vegetação.

Analisando os dados, pode-se constatar a existência de uma correlação negativa entre a distribuição das diferenças de vegetação e solo. Tal correlação é explicada naturalmente pelo consequente aumento na fração de solo resultante da diminuição da fração vegetação e vice-versa.

Outro fato a ser analisado na confecção da figura 2 é que ela é gerada, além das características intrínsecas das classes vegetação e solo, pela ocorrência de ruídos (comuns a esse tipo de imagem) e também de pequenas distorções entre as variáveis solo e vegetação ocasionadas pelo efeito da componente água/sombra. Entretanto, a ocorrência dessa distorção não afeta o processo de detecção de mudança para a imagem, e é esperado que os pixels que não sofreram alteração entre as duas datas (classe não-mudança) tendam a ocupar uma região próxima ao centro do diagrama de dispersão (inserida no círculo da figura 2), enquanto $p i-$ xels que sofreram alterações tendem a se deslocar para uma das duas extremidades do diagrama de dispersão dos dados, dependendo do tipo de mudança ocorrido nesses pixels (região de solo mudando para vegetação ou vice-versa).

Os dados de imagens de diferenças das frações vegetação e solo em imagens de sensoriamento remoto em geral tendem a apresentar um comportamento semelhante ao apresentado na figura 2. Nesta, a dispersão dos dados da imagem de diferenças é apresentada em duas dimensões (diferença na fração vegetação e diferença na fra- ção solo). Todavia, em três dimensões, esses dados apresentam um acúmulo de pixels nas proximidades da origem dos eixos, como pode ser ilustrado na figura 3 , através de um modelo teórico do perfil da dispersão de dados, no qual é mostrado um esboço dos perfis dos histogramas dos pixels referentes às classes mudança e não-mudança.

Na figura 3, pode ser vista a existência de uma sobreposição entre essas duas classes. Supõe-se, a partir da análise da figura 3 , que ambos os perfis dos histogramas apresentam uma distribuição normal, bivariada (diferença na fração vegetação e diferença na fração solo). Ademais, o histograma da classe mudança apresenta uma dispersão grande, com média próxima à origem, e a classe não-mudança uma dispersão pequena, quase centrada na origem, o que corrobora a ideia intuitiva de que pixels sem mudança significativa devam estar próximos de zero, enquanto pixels representando mudança assumem poucos valores nesse intervalo.

No caso em particular da imagem diferença que está sendo estudada neste trabalho, uma análise no histograma de frequência dos pixels desta imagem diferença mostra que uma quantidade muito grande de pixels está próxima à origem dos eixos diferença vegetação e solo. 0 centro desse agrupamento de pixels, porém, não está centrado na origem dos eixos, mas apresentando um pequeno deslocamento no sentido positivo do eixo diferença fração solo e praticamente centrado na origem em relação ao eixo diferença fração vegetação, isto é, centrado próximo ao valor 0 para a diferença fração vegetação e 0,1 para a diferença fração solo, como pode ser visto na figura 4 .

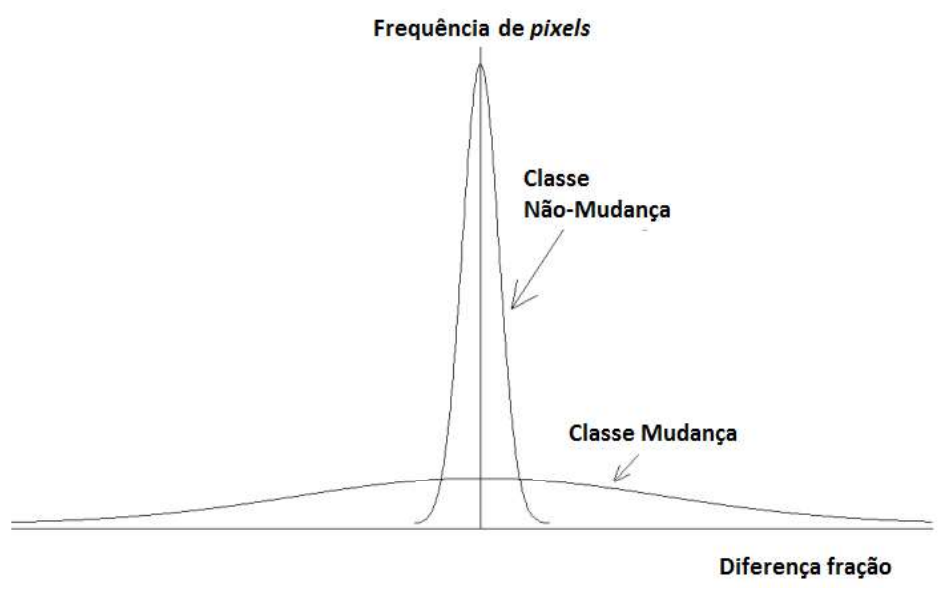

Figura 3. Distribuições das classes mudança e não-mudança.

Figure 3. Distributions of the change and no-change classes. 


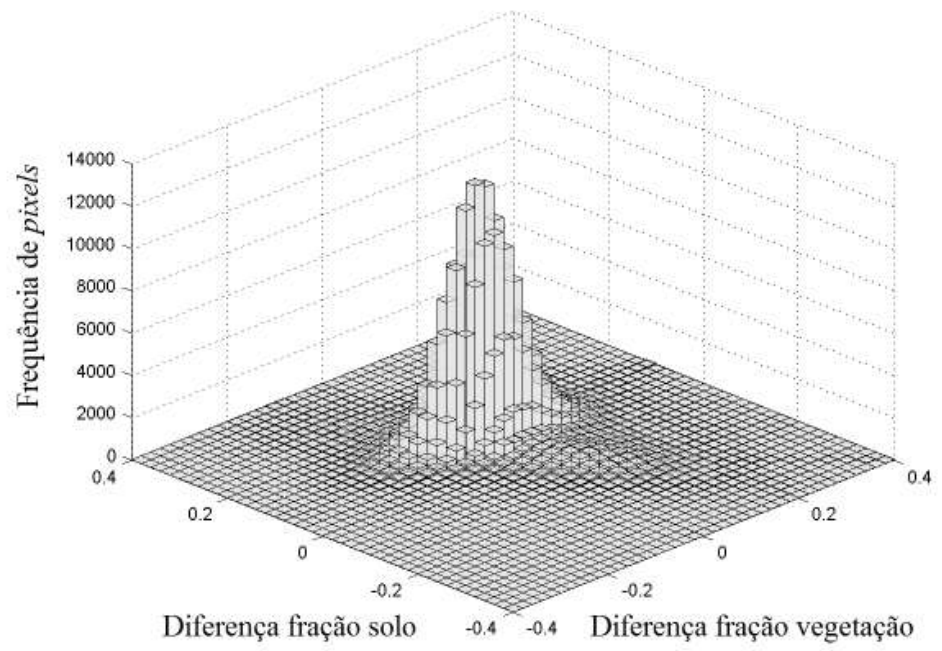

Histograma de frequência de pixels da imagem diferença

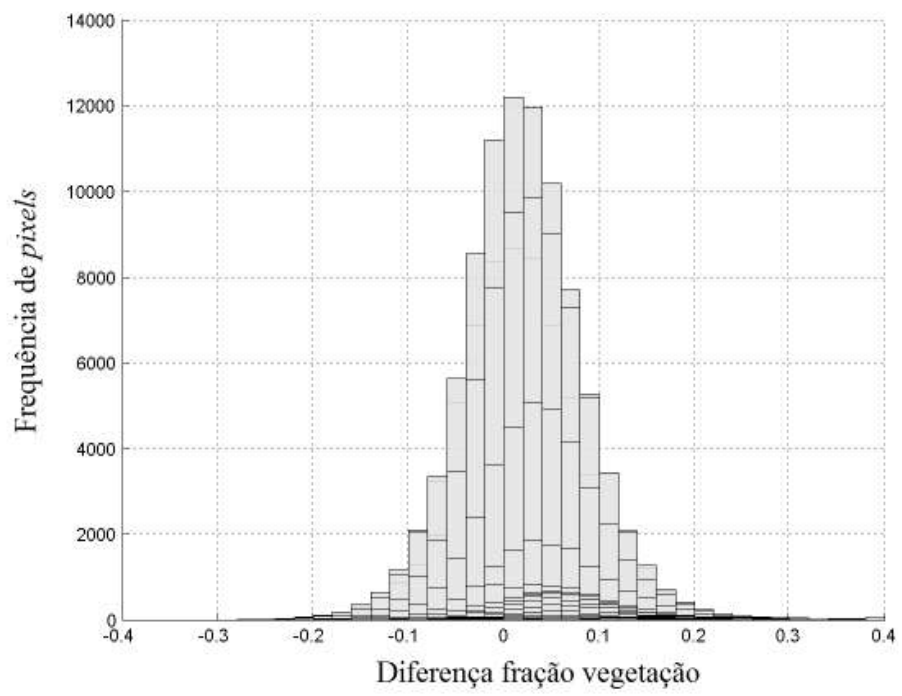

$\mathrm{C}$

Histograma de frequência de pixels da imagem diferença

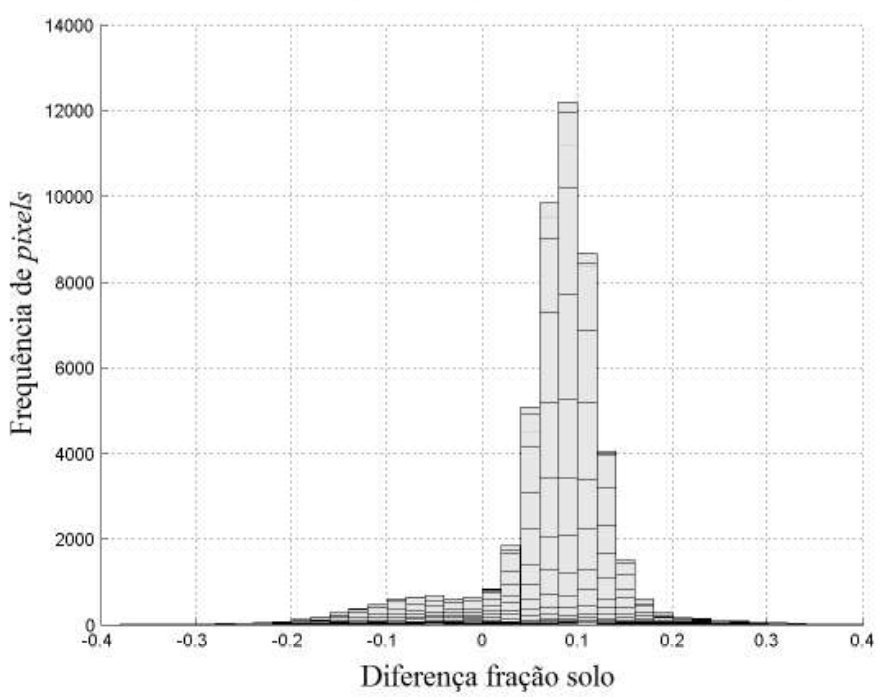

Figura 4. Histogramas. A) Histograma de frequência de pixels da imagem diferença; B) Perfil do histograma de frequência sobre o eixo diferença fração vegetação; C) Perfil do histograma de frequência sobre o eixo diferença fração solo.

Figure 4. Histogram. A) Histogram of frequency of pixels of the difference image; B) Profile of the histogram of frequency on the axis difference fraction vegetation; $C$ ) Profile of the histogram of frequency on the axis difference fraction soil. 
Como o histograma da figura 4 mostra que a distribuição de pixels ao longo dos eixos diferença vegetação e solo é semelhante ao que teoricamente é apresentado nas figuras 2 e 3 , isto é, elas apresentam distribuição normal bivariada, então o algoritmo EM pode ser usado para fins de estimação dos parâmetros (vetor de médias, matriz de covariâncias e probabilidade a priori) dessas duas distribuições (Duda et al., 2001). Além disso, como também os valores dos parâmetros deste estudo são muito próximos dos teoricamente esperados, pode-se, pela robustez do método EM, utilizar os parâmetros teóricos supostos nas figuras 2 e 3 como valores iniciais no algoritmo EM. Sendo assim, além de se considerar que ambas as distribuições sejam normais, com médias na origem, admite-se que a diferença entre as duas distribuições vai se concentrar nas variâncias, com a classe mudança apresentando uma dispersão grande e a classe não-mudança evidenciando uma dispersão pequena, conforme mostra a figura 3 .

Destarte, consideram-se como valores iniciais do algoritmo EM as classes $\omega 1$ e $\omega 2$, respectivamente classes mudança e não-mudança. $E$, ainda, $\mathrm{M} 1, \mathrm{~S} 1, \mathrm{P}(\omega 1), \mathrm{M} 2, \mathrm{~S} 2, \mathrm{P}(\omega 2)$ representam os vetores de médias, matrizes de covariâncias e probabilidades a priori de cada classe. Finalmente, para esses valores iniciais, algumas considerações são necessárias e podem ser ilustradas por meio da figura 1.

A classe mudança apresenta uma distribuição bivariada, alongada na direção de maior dispersão, que é estimada pelo primeiro autovalor ( $\lambda 1$ ). Para valor inicial no EM, propõe-se utilizar a matriz de covariância para a totalidade das amostras de diferenças nas frações. Já as amostras da classe não-mudança estão concentradas em torno da origem, apresentando uma pequena dispersão, causada pelos inevitáveis ruídos nos dados. Tal dispersão será estimada pela variância na direção ortogonal à distribuição de maior variação, ou seja, na direção do segundo autovetor. Essa variância é estimada pelo segundo autovalor ( $\lambda 2$ ). A distribuição será, portanto, circular em torno da origem com um raio proporcional a $\lambda 2$ (Zanotta \& Haertel, 2012).

Como geralmente o número de pixels apresentando pequena mudança ou mudança nula é muito maior do que o número de pixels com sinais claros de mudança. E, considerando a robustês do método EM, os valores iniciais das probabilidades a priori podem ser estimados inicialmente como 0,1 para mudança e 0,9 para não-mudança, uma vez que essa diferença exprime aproximadamente o comportamento dos dados.

Assim, as estimativas iniciais para cada classe podem ser determinadas pela equação 3 .

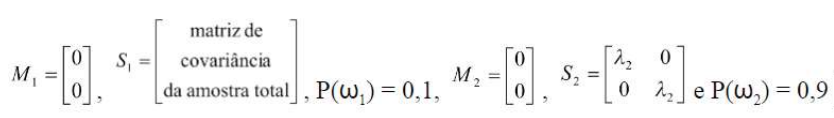

(Equação 3).

Após a convergência do algoritmo EM, os parâmetros obtidos foram utilizados na obtenção de amostras de treinamento para a classificação com SVM.

\subsubsection{SVM com amostragem normal multivariada}

A classificação com SVM utilizada neste trabalho está baseada em Abe (2005), cujo problema pode ser expresso pelas equações 4 e 5 .

Maximizar:

$$
\sum_{i=1}^{M} \alpha_{i}-\frac{1}{2} \sum_{i, j=1}^{M} \alpha_{i} \alpha_{j} y_{i} y_{j} K\left(\mathbf{x}_{i}, \mathbf{x}_{j}\right) \quad \text { (Equação 4) }
$$

Sujeito a

$$
\sum_{i=1}^{M} \alpha_{i} y_{i}=0 \text { e } C \geq \alpha_{i} \geq 0 \text {, para } \mathrm{i}=1, \ldots, \mathrm{M}
$$

(Equação 5)

Onde: $\alpha$ i são multiplicadores de Lagrange, $\mathrm{M}$ é o número de amostras consideradas, $\mathrm{K}(\mathrm{xi}, \mathrm{xj})$ é a função de kernel e C é o parâmetro de margem ou constante de custo.

Neste trabalho, foram utilizados o kernel polinomial e o kernel Gaussiano - ou kernel RBF (Radial Basis Function) ou Kernel de Função de Base Radial -, descritos, respectivamente, pelas equações 6 e 7 .

$$
\begin{array}{ll}
K\left(\boldsymbol{x}, \boldsymbol{x}^{\prime}\right)=\left(\boldsymbol{x}^{T} \boldsymbol{x}^{\prime}\right)^{d} & \text { (Equação 6) } \\
K\left(\boldsymbol{x}, \boldsymbol{x}^{\prime}\right)=e^{-\gamma\left|x-x^{\prime}\right|^{2}} & \text { (Equação 7) }
\end{array}
$$


Os algoritmos utilizados para a implementação do SVM foram desenvolvidos em MATLAB. Para fins de treinamento do classificador SVM, foram utilizadas amostras randômicas extraídas a partir de uma distribuição normal multivariada, obtidas por meio da função mvnrnd.m do MATLAB, usando em cada caso os parâmetros da respectiva distribuição estimados via EM.

Os experimentos com SVM foram feitos com os kernels polinomial e RBF com 200 e 400 amostras de treinamento para cada classe, utilizando as equações 4, 5, 6 e 7 .

\section{Resultados}

Conforme mencionado anteriormente, os experimentos foram realizados com duas imagens Landsat 5-TM cobrindo a mesma área, localizada no Estado de Roraima. A imagem da primeira data foi adquirida em outubro de 1991, e a imagem da segunda data, em abril de 1994. Nos experimentos, foram consideradas apenas as frações resultantes para vegetação e solo. As imagens utilizadas possuem 390.625 pixels distribuídos em um quadrado de 625 por 625 pixels.

Para a avaliação da acurácia dos resultados obtidos neste trabalho, foram utilizados dois procedimentos: a análise qualitativa, realizada por meio da produção do mapa de mudança após cada classificação, e a análise quantitativa, realizada a partir da construção da matriz de confusão.

Como a avaliação quantitativa necessita de verdade terrestre para a construção da matriz de confusão, foram realizados dois tipos de experimentos: um com imagem sintética e outro com imagem real.

A maior utilidade da construção de uma imagem sintética reside na possibilidade do conhecimento das alterações ocorridas. A imagem sintética foi produzida por meio da inclusão de alterações controladas na imagem Landsat-TM empregada como imagem da data 1 .

No caso do problema da detecção de mudanças, a matriz de confusão apresenta o formato abaixo, descrito no quadro 1 , em que as colunas representam a verdade terrestre e as linhas, a classificação obtida.
Quadro 1. Matriz de Confusão.

Chart 1. Matrix of Confusion.

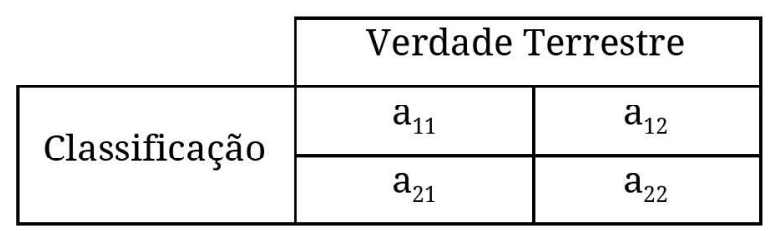

Considerando a classe 1 como mudança e a classe 2 como não-mudança, os termos da matriz podem ser interpretados da seguinte maneira: $a_{11}$ representa a quantidade de amostras da classe mudança que foram classificadas como mudança; $a_{12}$ representa a quantidade de amostras da classe não-mudança que foram classificadas como mudança; $a_{21}$ representa a quantidade de amostras da classe mudança que foram classificadas como não-mudança; e $\mathrm{a}_{22}$ representa a quantidade de amostras da classe não-mudança que foram classificadas como não-mudança.

Radke et al. (2005) definem algumas medidas que podem ser extraídas da matriz de confusão. A seguir estão descritas as que foram utilizadas neste trabalho.

Taxa de Falsos Alarmes - representa a proporção de pixels que não sofreram mudanças, mas foram classificados na classe mudança. Pode ser estimada pela equação 8 .

$$
F A=\frac{a_{12}}{a_{11}+a_{12}} \quad \text { (Equação 8) }
$$

Taxa de Detecção ou Acurácia do Usuário para a Classe Mudança - representa a proporção de pixels classificados como mudança entre aqueles que são realmente pixels de mudança. Pode ser estimada pela equação 9 .

$$
A U=\frac{a_{11}}{a_{11}+a_{21}} \quad \text { (Equação 9) }
$$

As mudanças aplicadas sinteticamente correspondem ao simples transporte de algumas áreas para outras regiões da imagem. 0 objetivo da construção dessa imagem foi selecionar amostras de teste para as classes mudança e não-mudança. Utilizando a imagem de data 1 selecionaram-se duas regiões. A primeira, de vegetação, foi transportada para um local próximo, também de vege- 
tação, para que posteriormente fossem selecionadas amostras de teste para a classe não-mudança. A segunda, de vegetação, foi transportada para um local de solo para que posteriormente fossem selecionadas amostras de teste para a classe mudança, conforme mostra a figura 5 .

As regiões utilizadas na construção da imagem sintética foram quadrangulares, contendo cada uma um total de 900 pixels. Posteriormente, foram selecionados, de cada uma das regiões, conjuntos com 200, 300 e 400 amostras de teste para cada classe, sendo que da região 1 foram selecionadas as amostras para a classe não-mudança e da região 2 amostras da classe mudança.
Nos testes com dados reais, as imagens de diferença foram obtidas pela diferença pixel a pixel entre a imagem fração da data 2 (1994) e a imagem fração da data 1 (1991).

A figura 6 mostra a imagem de diferenças produzida nos testes com a imagem real.

Nos testes com dados sintéticos, as imagens de diferença foram obtidas pela diferença pixel a pixel entre a imagem fração alterada sinteticamente e a imagem fração original da data 1.

A imagem sintética foi utilizada apenas para a seleção de amostras de teste. Todo o processo de seleção de amostras de treinamento foi realizado com as imagens reais.
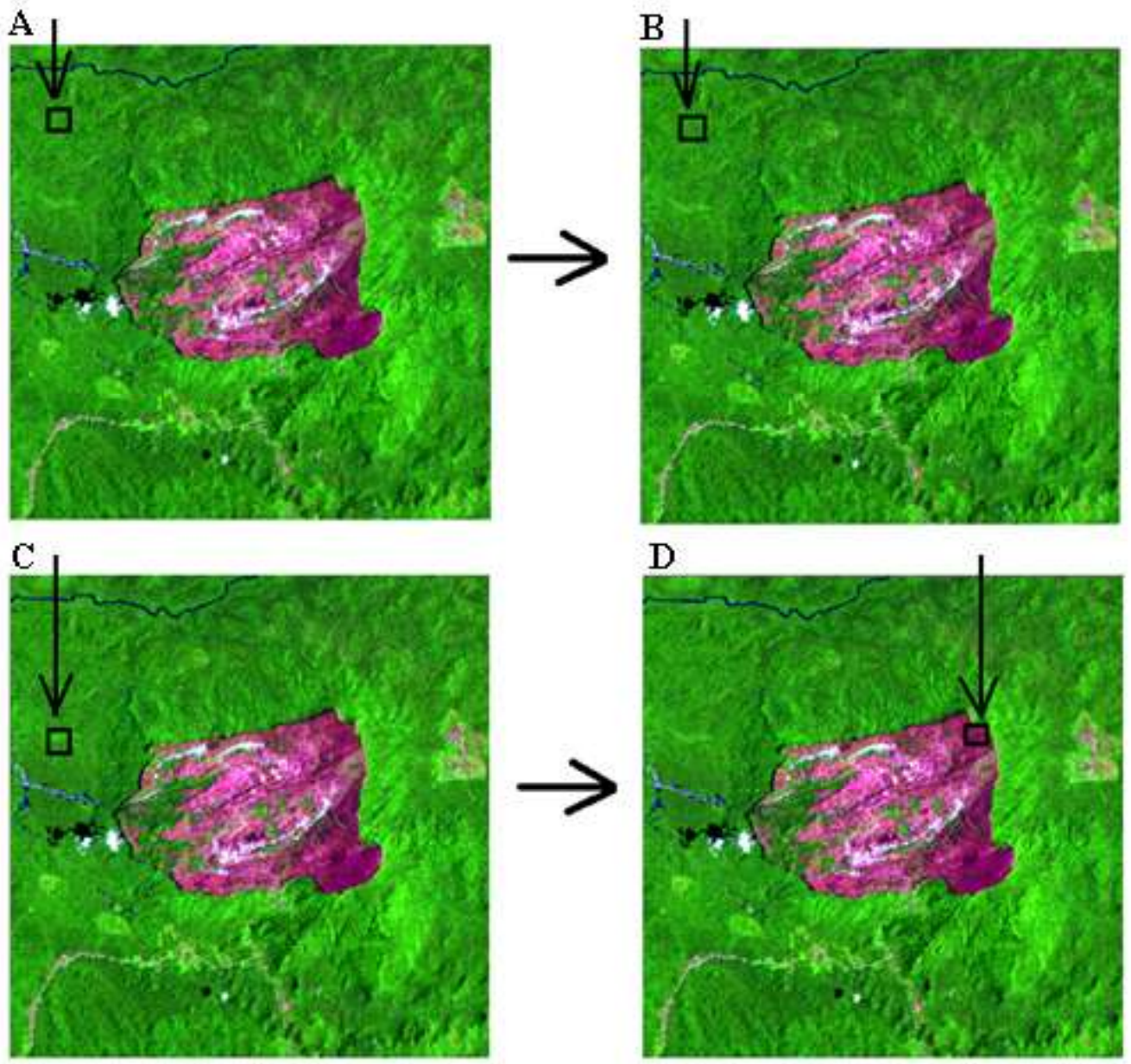

Figura 5. Mudanças sintéticas produzidas da imagem. A) Região de vegetação copiada para ser colada em outra região de vegetação; B) Região de vegetação colada em outra região de vegetação; C) Região de vegetação copiada para ser colada em uma região de solo. D) Região de vegetação colada em uma região de solo.

Figure 5. Synthetic changes produced from the image. A) Region of vegetation copied to be pasted in another region of vegetation; B) Region of vegetation glued in another region of vegetation; C) Vegetation region copied to be pasted in a soil region. D) Region of vegetation glued to a soil region. 


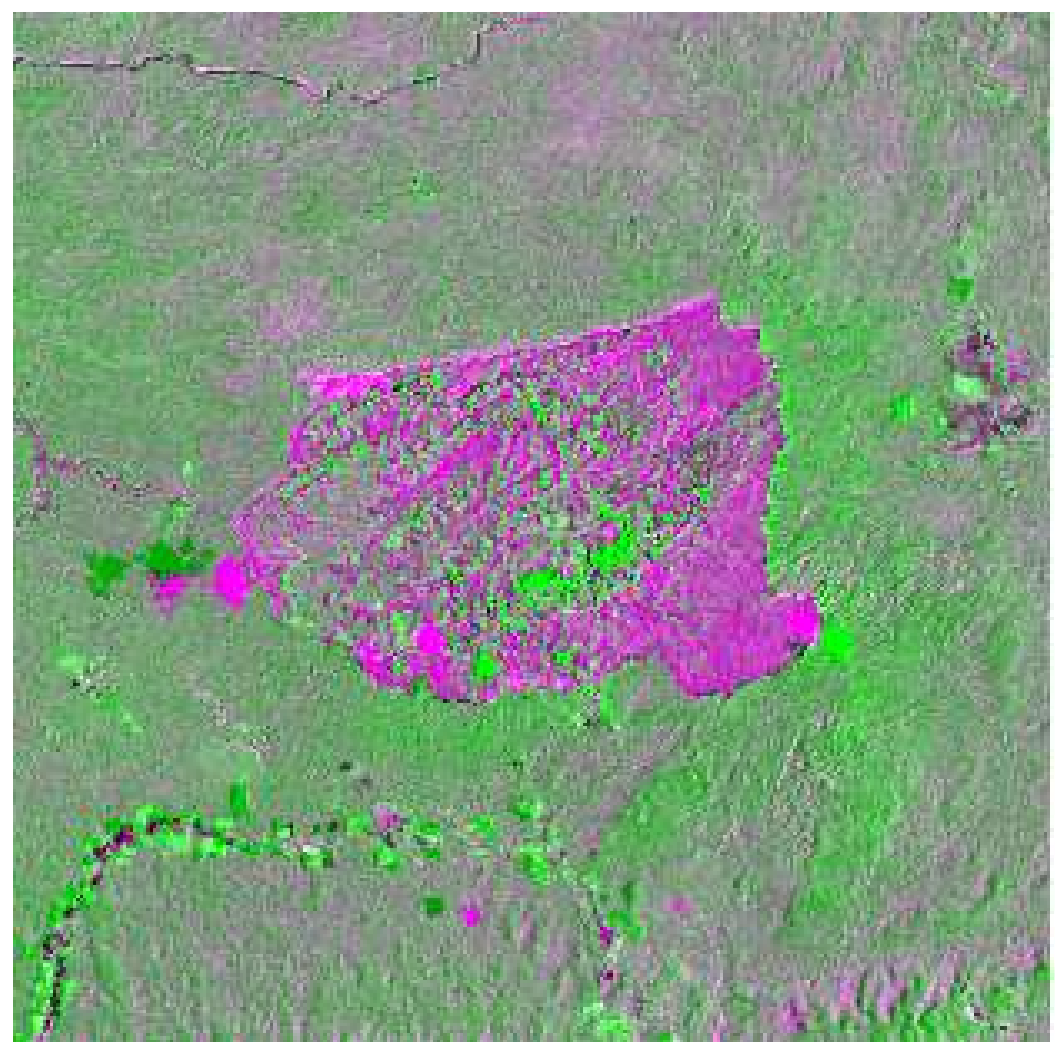

Figura 6. Imagem de diferença com dados reais. Composição em falsa cor 3(R) 2(G) 3(B). Figure 6. Difference image with real data. False color composition $3(R) 2(G) 3(B)$.

\subsection{Testes com a imagem sintética}

Em todos os experimentos foram realizados testes com 200 e 300 amostras de treinamento para cada classe e 200, 300 e 400 amostras de teste para cada classe. As amostras de teste foram selecionadas conforme descrito anteriormente. No caso do kernel polinomial, para o parâmetro d (grau do polinômio), foram realizados testes com d variando de 2 a 5 . 0 valor utilizado para o parâmetro $C$ foi 10 , por ter apresentado bons resultados em trabalhos anteriores (Andreola, 2009).
No caso do kernel RBF, para o parâmetro $\gamma$, foram utilizados 1, 2, 5 e 10; para o parâmetro C, também igual a 10.

As tabelas 1 e 2 mostram a acurácia obtida com o uso de cada kernel para 200 e 300 amostras de treinamento, com destaque para os parâmetros em que se obteve a melhor acurácia. As tabelas mostram a taxa de falsos alarmes e a acurácia do usuário (ou taxa de detecção).

Como as acurácias do usuário foram semelhantes, para a escolha dos parâmetros que obtiveram os melhores resultados, observaram-se os que produziram as menores taxas de falsos alarmes.

Tabela 1. Acurácia do SVM polinomial utilizando a imagem sintética e 300 amostras de teste. Table 1. Accuracy of the polynomial SVM using the synthetic image and 300 test samples.

\begin{tabular}{cccc}
\hline $\begin{array}{c}\text { Número de Amostras } \\
\text { de Treinamento }\end{array}$ & Grau & $\begin{array}{c}\text { Acurácia do Usuário para } \\
\text { a Classe Mudança }\end{array}$ & $\begin{array}{c}\text { Taxa de Falsos } \\
\text { Alarmes }\end{array}$ \\
\hline $\mathbf{2 0 0}$ & $\mathbf{2}$ & $\mathbf{9 6 , 3} \%$ & $\mathbf{1 , 3 \%}$ \\
300 & 2 & $96,3 \%$ & $2 \%$ \\
200 & 3 & $96 \%$ & $1,3 \%$ \\
300 & 3 & $96 \%$ & $3 \%$ \\
200 & 4 & $95,7 \%$ & $1,7 \%$ \\
300 & 4 & $96 \%$ & $20 \%$ \\
200 & 5 & $94,7 \%$ & $2,3 \%$ \\
300 & 5 & $96 \%$ & $15,3 \%$ \\
\hline
\end{tabular}


Tabela 2. Acurácia do SVM RBF utilizando a imagem sintética e 300 amostras de teste. Table 2. Accuracy of the SVM RBF using the synthetic image and 300 test samples.

\begin{tabular}{cccc}
\hline $\begin{array}{c}\text { Número de Amostras } \\
\text { de Treinamento }\end{array}$ & $\boldsymbol{r}$ & $\begin{array}{c}\text { Acurácia do Usuário } \\
\text { para a Classe Mudança }\end{array}$ & Taxa de Falsos Alarmes \\
\hline 200 & 1 & $97 \%$ & $5,3 \%$ \\
300 & 1 & $94,7 \%$ & $2,3 \%$ \\
200 & 2 & $96 \%$ & $2,7 \%$ \\
300 & 2 & $97 \%$ & $5,3 \%$ \\
200 & 5 & $96 \%$ & $\mathbf{1 , 7 \%}$ \\
300 & 5 & $97 \%$ & $4 \%$ \\
200 & 10 & $97,7 \%$ & $28,7 \%$ \\
300 & 10 & $97,3 \%$ & $24 \%$ \\
\hline
\end{tabular}

\subsection{Testes com a imagem real}

Em todos os experimentos, foram realizados testes com 200 e 300 amostras de treinamento para cada classe. Os parâmetros utilizados aqui foram os mesmos dos testes com a imagem sintética, ou seja, em ambos os casos o valor utilizado para a variável $C$ foi 10 ; no caso do kernel polinomial, para o parâmetro $d$, grau do polinômio, foram realizados testes variando de 2 a 5; no caso do kernel RBF, para o parâmetro $\gamma$, foram utilizados os valores $1,2,5$ e 10.
As figuras 7 e 8 mostram os mapas de mudança para os kernels polinomial e RBF, comparando os testes com 200 e 300 amostras de treinamento, onde os pontos pretos representam os pixels classificados como mudança.

Observando-se os mapas referentes aos parâmetros que obtiveram as melhores acurácias nos testes com a imagem sintética, é possível constatar que, nos testes com a imagem real, os mapas produzidos para o kernel RBF são semelhantes para os casos de 200 e 300 amostras de treinamento, o que não ocorre com o polinomial, em que há uma diferença nos graus 4 e 5 .

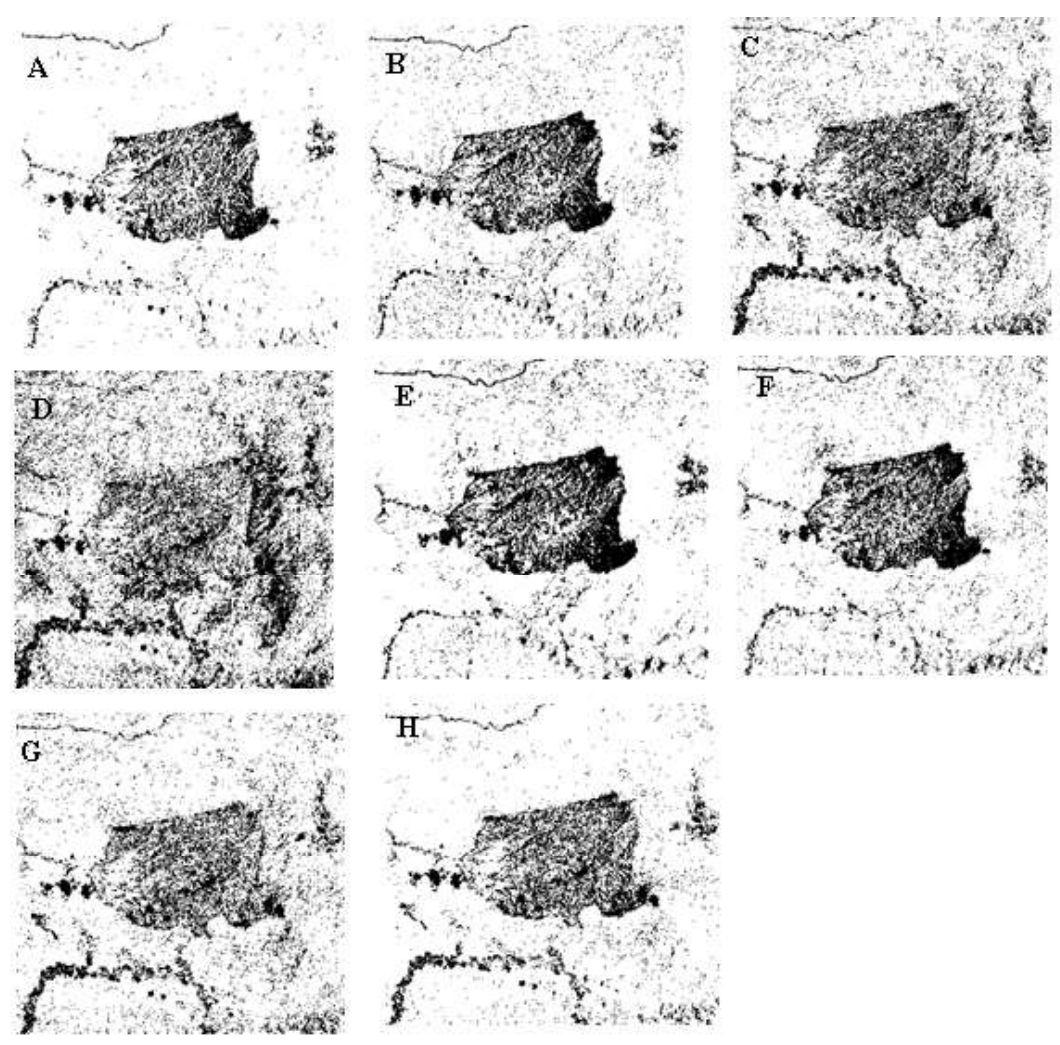

Figura 7. Mapa de mudança no experimento com dados reais com kernel polinomial. A) d = 2 e 200 amostras; B) d = 3 e 200 amostras; C) d = 4 e 200 amostras; D) d = 5 e 200 amostras; E) d = 2 e 300 amostras; F) d = 3 e 300 amostras; G) d = 4 e 300 amostras; $\mathrm{H}) \mathrm{d}=5$ e 300 amostras.

Figure 7. Change map in experiment with real data with polynomial kernel. A) $d=2$ and 200 samples; $B$ ) $d=3$ and 200 samples; $C$ ) $d=4$ and 200 samples; D) $d=5$ and 200 samples; $E) d=2$ and 300 samples; F) $d=3$ and 300 samples; $G$ ) $d=4$ and 300 samples; H) $d=5$ and 300 samples. 


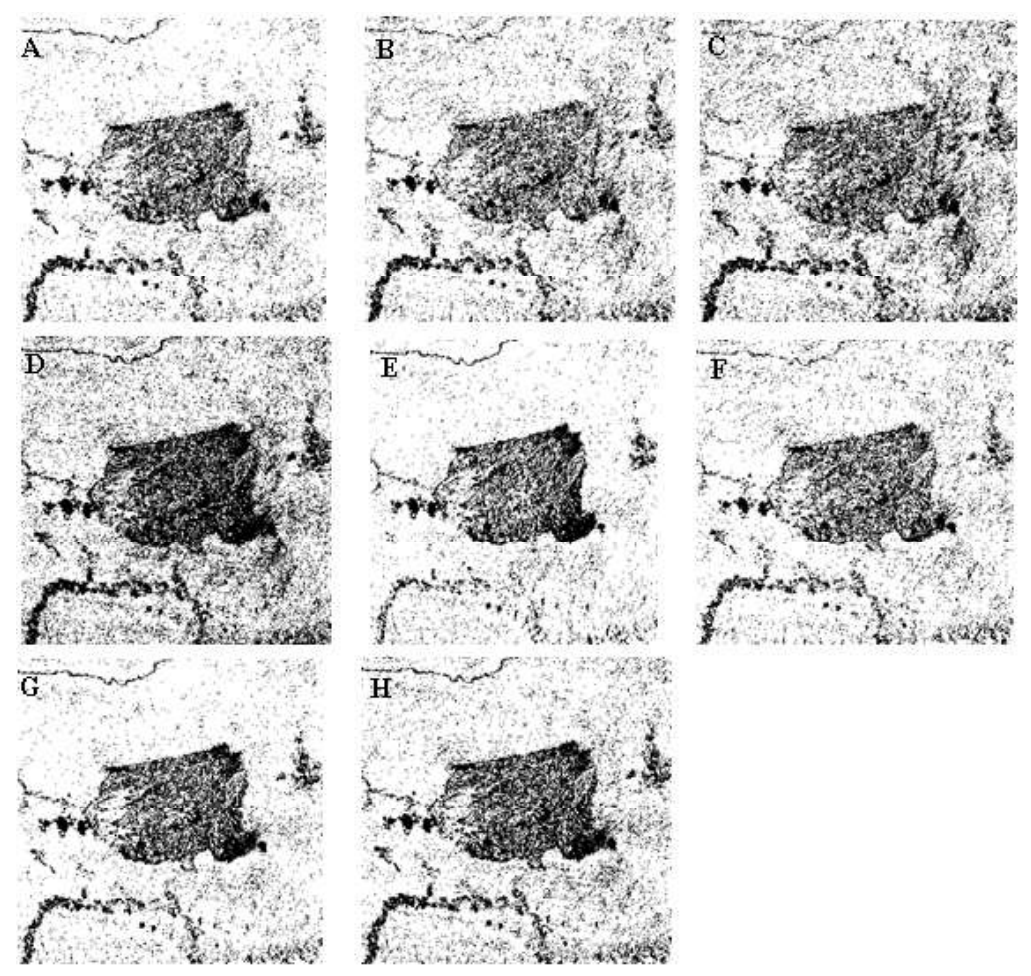

Figura 8. Mapa de mudança no experimento com dados reais com kernel RBF. A) $\gamma=1$ e 200 amostras; B) $\gamma=2$ e 200 amostras; C) $\gamma=5$ e 200 amostras; D) $\gamma=10$ e 200 amostras; E) $\gamma=1$ e 300 amostras; F) $\gamma=2$ e 300 amostras; G) $\gamma=5$ e 300 amostras; H) $\gamma=10$ e 300 amostras.

Figure 8. Change map in the experiment with real data with RBF kernel. A) $\gamma=1$ and 200 samples; B) $\gamma=2$ and 200 samples; C) $\gamma$ $=5$ and 200 samples; D) $\gamma=10$ and 200 samples; $E) \gamma=1$ and 300 samples; F) $\gamma=2$ and 300 samples; $G$ ) $\gamma=5$ and 300 samples; $\mathrm{H}$ ) $\gamma=10$ and 300 samples.

\section{Discussão dos resultados}

Foram apresentados dois tipos de experimentos: um com imagens sintéticas e outro com imagens reais. Tanto no primeiro tipo de experimento quanto no segundo foram realizados testes com SVM com os kernels polinomial e RBF. O experimento com dados sintéticos teve como objetivo testar o processo com um par de imagens onde se tinha um conhecimento das alterações existentes.

Para a avaliação da acurácia dos resultados obtidos neste trabalho foram utilizados dois procedimentos: análise qualitativa, realizada por meio da produção do mapa de mudança após cada classificação no experimento com dados reais e análise quantitativa, realizada por meio da análise da matriz de confusão no experimento com dados sintéticos.

Nos experimentos com a imagem sintética, foi possível observar que o kernel RBF tem os resultados muito semelhantes para todos os conjuntos de amostras de teste, independente do tamanho do conjunto de amostras de treinamento, para valores de gama 5 ou 10, bem como apresentou a menor taxa de falsos alarmes. No caso do polino- mial, os resultados são semelhantes para os graus 2 e 3 .

Esse fato pode ser observado nas curvas de acurácias globais das figuras 9 e 10, construídas a partir dos resultados com experimentos utilizando 200 e 300 amostras de treinamento e conjuntos de 200, 400 e 300 amostras de teste.

Embora os parâmetros com melhor acurácia tenham sido distintos em cada teste, é possível verificar que, no caso do kernel polinomial, o grau 2 foi melhor em dois dos testes e o kernel RBF com $\gamma$ igual a 5 também foi melhor em dois; no outro teste, os resultados foram semelhantes. Dessa forma, foram considerados os melhores parâmetros.

$\mathrm{Na}$ avaliação qualitativa, os resultados foram semelhantes para todos os conjuntos de amostras de teste utilizados, em conformidade com trabalhos anteriores de outros autores. Nos testes com a imagem real, uma inspeção visual dos resultados obtidos revela uma boa concordância com os encontrados em Zanotta (2010), onde os pixels que apresentam maiores graus de pertinência à classe mudança correspondem aos classificados como mudança. Também evidenciam concordância com os trabalhos de Haertel et al. (2004), e Bittencourt 

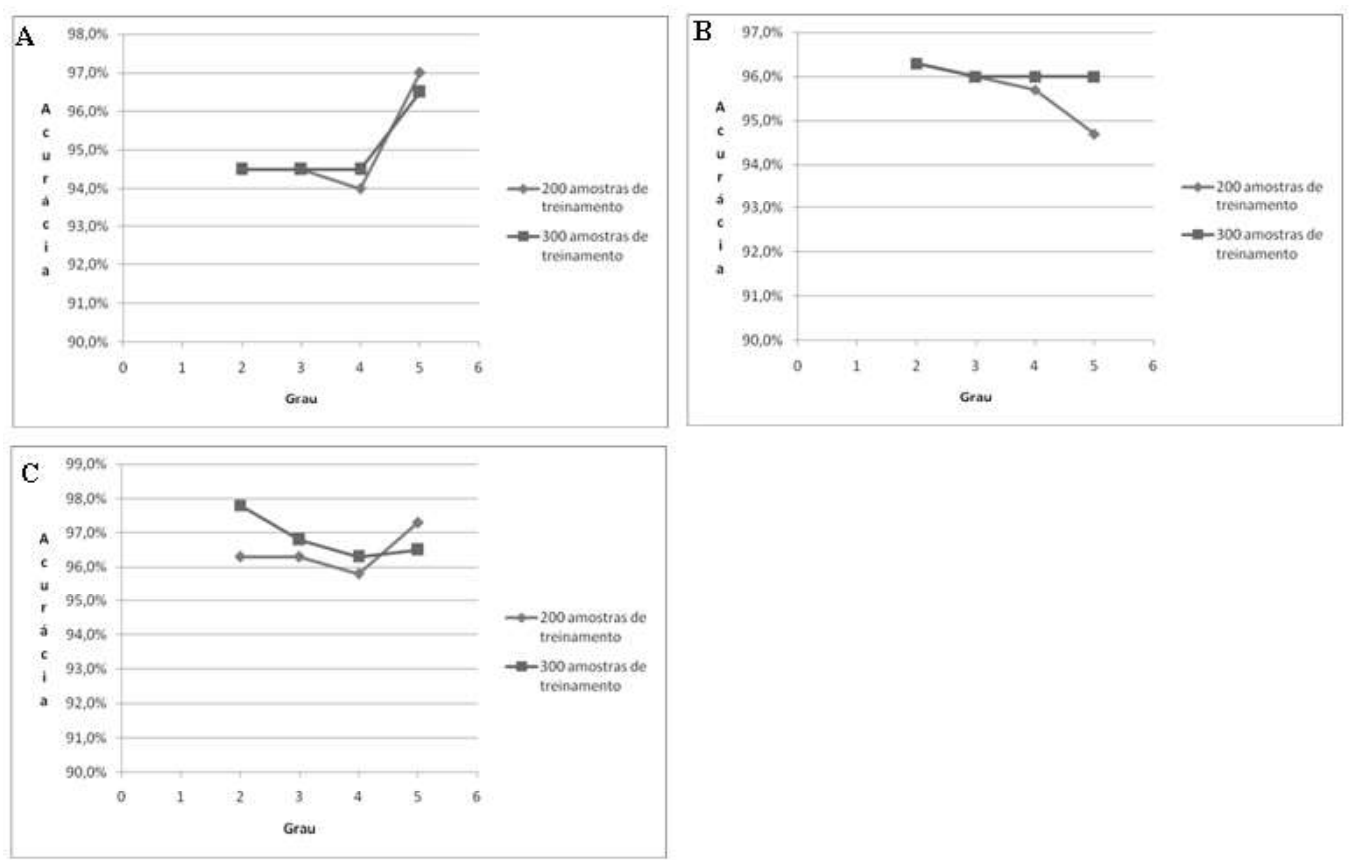

Figura 9. Acurácia global do SVM Polinomial nos testes com a imagem sintética com 200 e 300 amostras de treinamento. A) 200 amostras de teste; B) 300 amostras de teste; C) 400 amostras de teste.

Figure 9. Overall accuracy of the Polynomial SVM in the synthetic image tests with 200 and 300 training samples. A) 200 test samples; B) 300 test samples; C) 400 test samples.

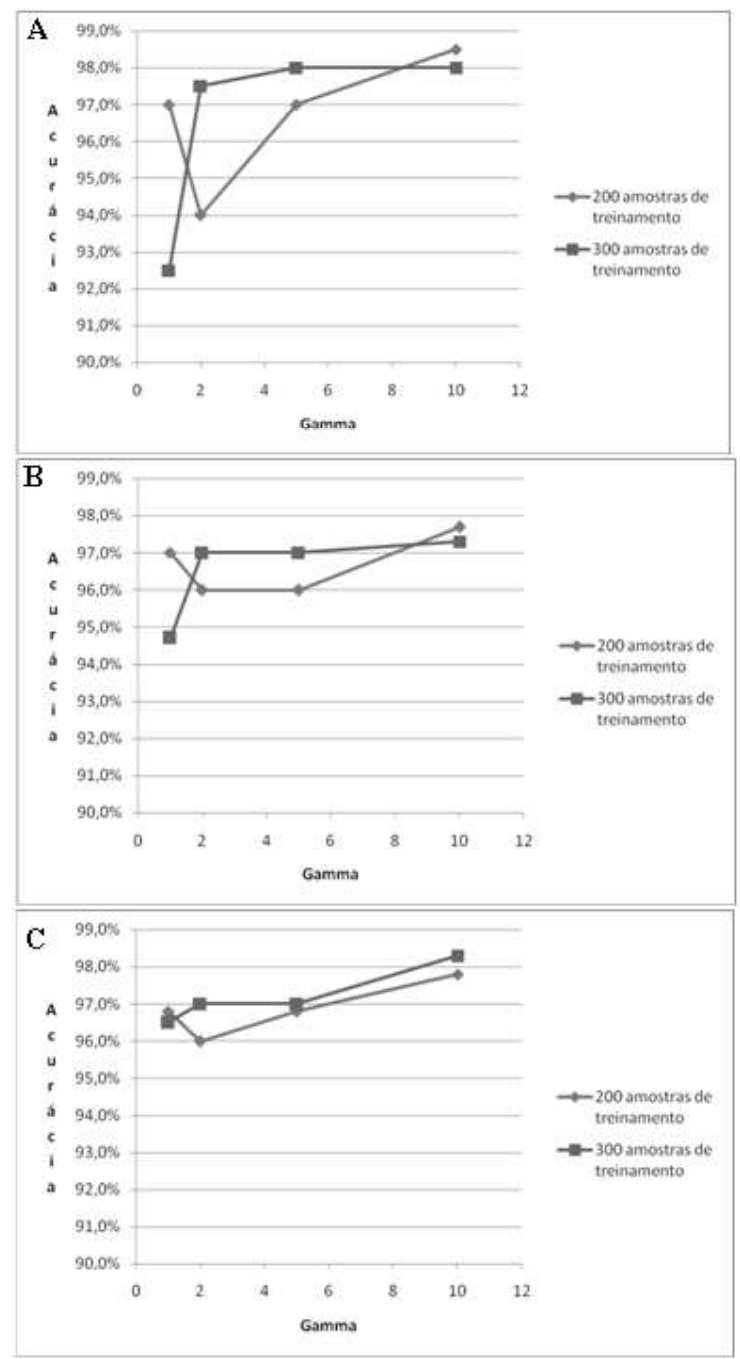

Figura 10. Acurácia global do SVM RBF nos testes com a imagem sintética com 200 e 300 amostras de treinamento. A) 200 amostras de teste; B) 300 amostras de teste; C) 400 amostras de teste.

Figure 10. Overall accuracy of the RBF SVM in the synthetic image tests with 200 and 300 training samples. A) 200 test samples; B) 300 test samples; C) 400 test samples. 
et al. (2013). Além disso, é possível verificar que, no caso do kernel polinomial, os mapas de mudança foram semelhantes nos testes com 200 e 300 amostras de treinamento, exceto nos graus 4 e 5 , o que também ocorreu nos testes com a imagem sintética.

Em relação aos resultados apresentados em Bittencourt et al. (2013), também percebe-se uma similaridade, embora naquele estudo se tenha uma preocupação com a eliminação dos falsos alarmes, os quais no presente estudo aparecem em menor quantidade do que os apresentados naquele trabalho, sem o processo de filtragem microbiológica proposto. Esta equivalência de resultados evidencia o bom desempenho do classificador SVM para detecção de mudanças com poucas amostras de treinamento.

Observando-se os mapas referentes aos parâmetros que obtiveram as melhores acurácias nos testes com a imagem sintética, é possível constatar que nos testes com a imagem real os mapas produzidos para o kernel RBF são semelhantes para os casos de 200 e 300 amostras de treinamento, o que não ocorre com o polinomial, em que há uma diferença nos graus 4 e 5 .

Ao comparar os dois tipos de kernels utilizados também foi possível observar que o mapa obtido no teste com o kernel polinomial de grau 2 e 200 amostras de treinamento e o obtido no teste com o kernel RBF $\operatorname{com} \gamma=1$ e 200 amostras de treinamento são semelhantes.

Acrescenta-se que, assim como em Zanotta (2010), o método proposto aqui visa detectar mudanças em ambientes onde há prevalecimento de vegetação e solo, tendo restrições para o uso em outros ambientes com diferentes componentes, principalmente pelas suposições utilizadas para a determinação dos valores iniciais do algoritmo EM. Sobre o EM, salienta-se que o processo converge para valores confiáveis desde que haja uma proporção razoável entre as duas distribuições, de forma que o método não alcançará a convergência se, em uma das duas classes, existirem um número muito superior de representantes (Ferreira, 2014).

Embora a metodologia proposta aqui tenha evidenciado resultados aceitáveis, cabe salientar que, em cenas naturais, as alterações ocorrem de uma forma gradual e contínua, o que torna difícil a definição objetiva de um limiar separando rigidamente a classe mudança da classe não-mudança. Destarte, sugere-se a investigação de abordagens que aliem as vantagens do classificador SVM, mostradas neste trabalho, a uma classificação em termos de graus de pertinência em cada classe, de modo que permitam que pixels pertençam, em parte, a mais de uma classe. Também se sugere o uso do SVM one-class, que tem sido utilizado em outras abordagens para o problema de detecção de mudanças envolvendo kernel, como o support vector data domain (Bovolo et al., 2010).

\section{Conclusões}

Os experimentos desenvolvidos neste trabalho mostram a adequação da metodologia proposta, produzindo resultados em média superiores a $94 \%$ de acurácia na detecção de alterações na cobertura do solo.

Como mencionado anteriormente, este trabalho investigou uma abordagem para detectar mudanças em ambientes onde há prevalecimento de vegetação e solo. Assim, sugere-se que o método seja investigado em cenas com outro tipo de componente, como, por exemplo, cenas urbanas.

Outro ponto a observar é que, embora esse trabalho tenha apresentado resultados semelhantes aos já existentes na área de detecção de mudanças, o fato de utilizar SVM indica que este é um método adequado para lidar com o problema da classificação de imagens hiperespectrais, uma vez que eles podem lidar com espaços de dimensões grandes de forma eficiente, lidam muito bem com amostras ruidosas e o modelo que define a fronteira de decisão é expresso como a função de um subconjunto de poucas amostras de treinamento em relação aos métodos bayesianos.

É importante salientar que em um método supervisionado a disponibilização de amostras de treinamento é geralmente uma tarefa de difícil execução e de custo elevado. Assim, o método proposto aqui pode ser vantajoso, uma vez que se mostrou eficiente mesmo com utilização de poucas amostras de treinamento, indicando que pode ser utilizado também com imagens hiperespectrais, sem a preocupação com o Fenômeno de Hughes (ABE, 2005) onde há uma degradação da acurácia a medida que o número de dimensões aumenta. 
Agradecimentos. Os autores agradecem ao Programa de Pós-Graduação em Sensoriamento Remoto (PPGSR) da UFRGS e a Vitor Haertel (in memoriam), uma vez que este trabalho está relacionado às teses de doutorado dos autores, desenvolvidas junto ao PPGSR.

\section{Referências bibliográficas}

Abe, S. 2005. Support Vector Machines for Pattern Classifications. Kobe, Springer, 343p.

Andreola, R. 2009. Support Vector Machines na classificação de imagens hiperespectrais. Porto Alegre, 130p. Dissertação de Mestrado, Programa de Pós-Graduação em Sensoriamento Remoto, Centro Estadual de Pesquisas em Sensoriamento Remoto e Meteorologia, Universidade Federal do Rio Grande do Sul.

Angelo, N.P. 2014. Uma abordagem para a detecção de mudanças em imagens multitemporais de sensoriamento remoto empregando Support Vector Machines com uma nova métrica de pertinência. Porto Alegre, 124p. Tese de Doutorado, Programa de Pós-Graduação em Sensoriamento Remoto, Centro Estadual de Pesquisas em Sensoriamento Remoto e Meteorologia, Universidade Federal do Rio Grande do Sul.

Bazi, Y., Bruzzone, L. \& Melgani, F. 2005. An unsupervised approach based on the generalized Gaussian Model to automatic change detection in multitemporal SAR images. IEEE Transactions on Geoscience and Remote Sensing, 43(4): 874-886.

Bittencourt, H.R., Saldanha, D.L. \& Haertel V. 2013. Multivariate approach to change detection from fraction images. Canadian Journal of Remote Sensing, 39 (3): 208-216.

Bovolo, F., Camps-Valls, G. \& Bruzzone, L. 2010. A Support Vector Domain Method for change detection in multitemporal images. Pattern Recognition Letters, 31(10): 1148-1154.

Bruzzone, L. \& Prieto, D.F. 2000. Automatic analysis of the difference image for unsupervised change detection. IEEE Transactions on Geoscience and Remote Sensing, 38(3): p. 1171-1182.

Bruzzone, L., Cossu, R. \& Vernazza, G. 2004. Detection of land-cover transitions by combining multidate classifiers. Pattern Recogniton Letters,
25(13): 1491-1500.

Camps-Valls, G. \& Bruzzone, L. 2005. Kernel-based methods for hyperspectral image classification. IEEE Transactions on Geoscience and Remote Sensing, 43(6): 1351-1362.

Castellana, L., D'addabbo, A. \& Pasquariello, G. 2007. A composed supervised/unsupervised approach to improve change detection from remote sensing. Pattern Recognition Letters, 28(4): 405-413.

Duda, R.O., Hart, P.E. \& Stork, D.G. 2001. Pattern classification. New York, John Wiley \& Sons, 680 p.

Ferreira, R.H.F. 2014. Uma abordagem para a detecção de mudanças em imagens multitemporais de sensoriamento remoto empregando Support Vector Machines. Porto Alegre, 89p. Tese de Doutorado, Programa de Pós-Graduação em Sensoriamento Remoto, Centro Estadual de Pesquisas em Sensoriamento Remoto e Meteorologia, Universidade Federal do Rio Grande do Sul.

Filippone, M. \& Sanguinetti, G. 2010. Information theoretic novelty detection. Pattern Recognition, 43(3): 805-814.

Haertel, V., Shimabukuro, Y.E. \& Almeida Filho, R. 2004. Fraction images in multitemporal change detection. International Journal of Remote Sensing, 10(23): 5473-5489.

Lu, D., Mausel, P., Brondizio, E. \& Moran, E. 2003. Change detection techniques. International Journal of Remote Sensing, 25(12): 2365-2407.

Malila, W.A. 1980. Change Vector Analysis: An Approach for Detecting Forest Changes with Landsat. In ANNUAL SYMPOSIUM ON MACHINE PROCESSING OF REMOTELY SENSED DATA SOIL INFORMATION SYSTEMS AND REMOTE SENSING AND SOIL SURVEY, 6, 1980, West Lafayette. Anais... West Lafayette, Purdue University, Laboratory for Applications of Remote Sensing, p. 326-335.

Radke, R.J., Andra, S.,Al-Kofahi, O. \& Roysam, B. 2005. Image change detection algorithms: a systematic survey. IEEE Transactions on Image Processing, 14(3): 294-307.

Shawe-Taylor, J. \& Cristianini, N. 2004. Kernel methods for pattern analysis. New York, Cambridge University Press, 477p.

Shimabukuro, Y. E. \& Smith, J. A. 1991. The least-squares mixing models to generate 
fraction images derived from remote sensing multispectral data. IEEE Transactions on Geoscience and Remote Sensing, 29(1): 16-20.

Singh, A. 1989. Digital change detection techniques using remotely-sensed data. International Journal of Remote Sensing, 43(10): 989-1003.

Zanotta, D. 2010. Uma abordagem fuzzy na detecção automática de mudanças do uso do solo usando imagens de fração e informações de contexto espacial. Porto Alegre, 88p. Dissertação de Mestrado, Programa de Pós-Graduação em Sensoriamento Remoto, Centro Estadual de Pesquisas em Sensoriamento Remoto e Meteorologia, Universidade Federal do Rio Grande do Sul.

Zanotta, D. \& Haertel, V. 2012. Gradual landcover change detection based on multitemporal fraction images. Pattern Recognition, 45: 29272937. 